\title{
Annual scientific meeting July 92014
}

\section{A CASE OF APPENDIX CARCINOID IN A PEDIATRIC PATIENT. A SUBTLE CLINICAL PRESENTATION}

\author{
E. Bindi, R. Angotti, A.L. Bulotta, M. Sica, E. Cerchia, F. Molinaro, M. Messina \\ Section of Pediatric Surgery, Department of Pediatrics, Obstetrics and Reproductive Medicine, University of Siena, Italy
}

\section{Introduction}

The appendix carcinoid, although rare (with an incidence of $0,08 \%)$, is the more frequent gastrointestinal tumor in children and teen. In most cases these tumors cannot be diagnosed or they can be incidentally found during a surgical exploration for acute abdomen. Only in few cases there are symptoms of a carcinoid's syndrome (flushing, diarrhea and wheezing). In this work we present the manegement of a patient with an appendix carcinoid revealed during an appendicectomy performed for a peritonitis caused by a perforated appendicitis, underlining the importance of diagnosis and long term follow-up.

\section{Case report}

A 13-years girl came to our attention with an history of ten days abdominal pain localized in the right iliac fossa. The clinical valutation, blood tests and abdominal ultrasound were suggestive of abdominal abscess and the patient undergone to surgical intervention. An appendicectomy was performed and the histological examination revealed the presence of a carcinoid localized on the top of the appendix. Actually, the patient is free from disease in a ten months follow-up.

\section{Conclusions}

Given occasional diagnosis and paucity of works in literature about the course of this kind of tumors in childhood, it would be preferable a conservative surgical treatment and a close long term follow-up.

\section{MANAGEMENT AND OUTCOME OF HERLYN-WERNER-WUNDERLICH SYNDROME: CONSERVATIVE TREATMENT IN PEDIATRIC AGE}

\author{
R. Angotti, A.L. Bulotta, E. Cerchia, E. Brandigi, F. Mariscoli, F. Molinaro, M. Messina \\ Section of Pediatric Surgery, Department of Pediatrics, Obstetrics and Reproductive Medicine, University of Siena, Italy
}

\section{Introduction}

Herlyn-Werner-Wunderlich syndrome (HWWS) is a rare congenital mullerian anomaly consisting of uterus didelphys, hemivaginal septum, and unilateral renal agenesis. Reported data in pediatric age are rare. We report our case of HWWS.

\section{Case report}

A 4-years-old girl came to our department with diagnosis of pelvic mass associated with lower abdominal pain since 1 year, that had worsened in the last 5 months. On physical examination the mass was palpated in the right lower abdomen. The external genitalia were normal. Abdominal ultrasound (US) showed a mass in the pelvic cavity associated with normal uterus and agenesis of right kidney. MRI with contrast confirmed all of those data and showed a transverse vaginal septum. Examination under anesthesia, cystoscopy and vaginoscopy were planned and done. Trans Hymenal resection of vaginal septum was performed. Postoperative course was normal. At last follow up (1 years after surgery) patient was in healthy.

\section{Discussion and Conclusions}

This syndrome is a rare anomaly in the spectrum of mullerian ducts diseases. Patients with HWWS usually are aymptomatic until adolescent age, but the onset can be earlier, as in our case. The suspicion must be considered in all females with renal agenesia. A correct diagnosis and management, indeed, ensure a good long term outcome without any problems in term of fertility and sexual aspects. 


\section{Annual scientific meeting October 272014}

THE HUMAN RACHIS: CAN IT BE CONSIDERED A SHOCK ABSORBER (THAT WAS PRODUCED BY EXAPTATION) RATHER THAN A COLUMN?

\section{Tanga, F. Ghelli, G. Gelati}

Corpus (International Group for the Cultural Studies of the body)

Our paper is focused on two fundamental points: the first one is a terminological proposal and the second one is a question. Obviously these two things are strictly related one another. The terminological proposal is aimed to name "vertebral shock absorber" the human rachis (globally considered, when it is in physiological conditions) in its most typical function: to sustain static/dynamic stresses, that moreover are directed according to its axial direction, obviously when this coincides with gravitational line. This aspects can be studied by modal analysis and by the model of Eigenvectors and Eigenvalues. According to our opinion, the mechanical feature must be considered as prevalent if compared with the structural one. Following it human rachis is usually named "column". This mechanic sustain is distributed on three lines that are summarily parallel and are linked one another (by isthmuses and vertebral arches to build an horizontal ring) so they can be considered a unique compact viscous-elastic system. Each one of these three vertical sub-structure is built as a stacking of metameric elements (modules) along a continuous line. This spatial linear disposition is regarding: vertebral bodies (that are alternated with inter-vertebral discs and are placed on the median line) and articular processes of vertebras (two symmetric lines posterior to vertebral bodies).

In chordates' notochord, in fishes' rachis and in the vertebral "beam" of tetrapods the solicitations are transversal and trend to disaggregate the vertebral chain. Instead, in human pilaster the main static solicitation, the gravitational charge, trends to make it collapse: the reciprocal pressure pushes the vertebras one against the others. Sagittal physiological curves seem a partial retreat under the gravitational charge, a beginning breaking of vertebral instable balance. Maybe it is so, but the sinuous shape of human rachis makes it much more resistant to further charges, both static (to lift something) and dynamic (falling down after an elevation jump). The curves elastically become more/less empathized and so they make the human rachis a very efficient shock absorber.

The related question is if we can define "exaptations" evolutionary jumps of rachis: from central axis of first fishes living in water to beam of terrestrial tetrapods, to shelf in first bipeds, to column or, as we propose, vertical shock absorber in human beings. In both cases, of a positive or of a negative answer, it could be opportune to establish criteria and limits to define an evolutionary transformation as "exaptation" or "not-exaptation".

The transformation of the function of the exoskeleton of arthropods (from protective shell in many water species to locomotion sustain in terrestrial species), of the feathers (from thermal defense in Dinosauria to aerodynamic structures in Birds) and of the appendixes of vertebrates (from fins in water species to paws in terrestrial tetrapods) have been considered exaptations. Similarly we propose of considering exaptation the change of mechanical function of human rachis.

Anyway every transformation is a change of function and/or of structure, but it could be heuristically interesting to have criteria and limits available to classify each case. 